\section{International Scientific Journal Theoretical \& Applied Science}

\author{
p-ISSN: 2308-4944 (print) e-ISSN: 2409-0085 (online) \\ Year: 2015 Issue: $01 \quad$ Volume: 21 \\ Published: $30.01 .2015 \quad$ http://www.T-Science.org
}

SECTION 2. Applied mathematics. Mathematical modeling.
Seytkhan Meldebekovich Koybakov

doctor of technical Sciences, Professor, academician of International Academy of Sciences of Ecology and Life Safety,

Pro-rector on scientific work and international relations, Taraz State University named after M.Kh. Dulati, Kazakhstan seithan@tarazinfo.kz

Sagat Zhunisbekov doctor of technical Sciences, Professor, Academician of the National Engineering Academy of the Republic of Kazakhstan, Academician of the Kazakhstan National Academy of Natural Sciences (KazNANS), rector Taraz technical Institute, Kazakhstan tar-ti@mail.ru

Alexandr Nikolayevich Shevtsov candidate of technical sciences, member of PILA (USA), corr.member of the KazNANS, president of IA TAS, department of «Mathematics», deputy director on Science of faculty of Information technologies, automation and telecommunications,

Taraz state University named after M.Kh. Dulati, Kazakhstan Shev_AlexXXXX@mail.ru

\title{
ABOUT MODEL AERODYNAMIC FLOW CHANNEL
}

Abstract: In this paper we study the dynamic characteristics of the process of the aerodynamic flow around the bed of the channel, in a flat non-stationary model of turbulent flow. The obtained data about the pressure and velocity at different speeds, and when the wind gusts up to $40 \mathrm{~m} / \mathrm{s}$.

Key words: wind, channel, flow, turbulence.

Language: Russian

Citation: Koybakov SM, Zhunisbekov S, Shevtsov AN (2015) ABOUT MODEL AERODYNAMIC FLOW CHANNEL. ISJ Theoretical \& Applied Science $01 \quad$ (21): 190-197. http://dx.doi.org/10.15863/TAS.2015.01.21.32

\section{ОБ ОДНОЙ МОДЕЛИ АЭРОДИНАМИЧЕСКОГО ОБТЕКАНИЯ КАНАЛА}

Аннотация: В данной работе исследуются динамические характеристики процесса аэродинамическо обтекания русла канала, в плоской нестационарной модели турбулентного потока. Получены данные о давлении и скорости обтекания при различных скоростях, а также при порывах ветра до 40 м/с.

Ключевые слова: ветер, канал, обтекание, турбулентность.

Большая часть территории Казахстана подвергается воздействию сильных ветров, вызывающих снежные и песчанные заносы. Водопроводящие каналы, эксплуатируемые в этих условиях, снижают пропускную способность вплоть до полной остановки. Причиной заноса - являются турбулентные потоки воздуха возникающие в русле канала. Рассмотрим математическую модель моделирующую поток воздуха, насыщенный снежными или песчанными частицами, набегающий на русло канала [1]. 


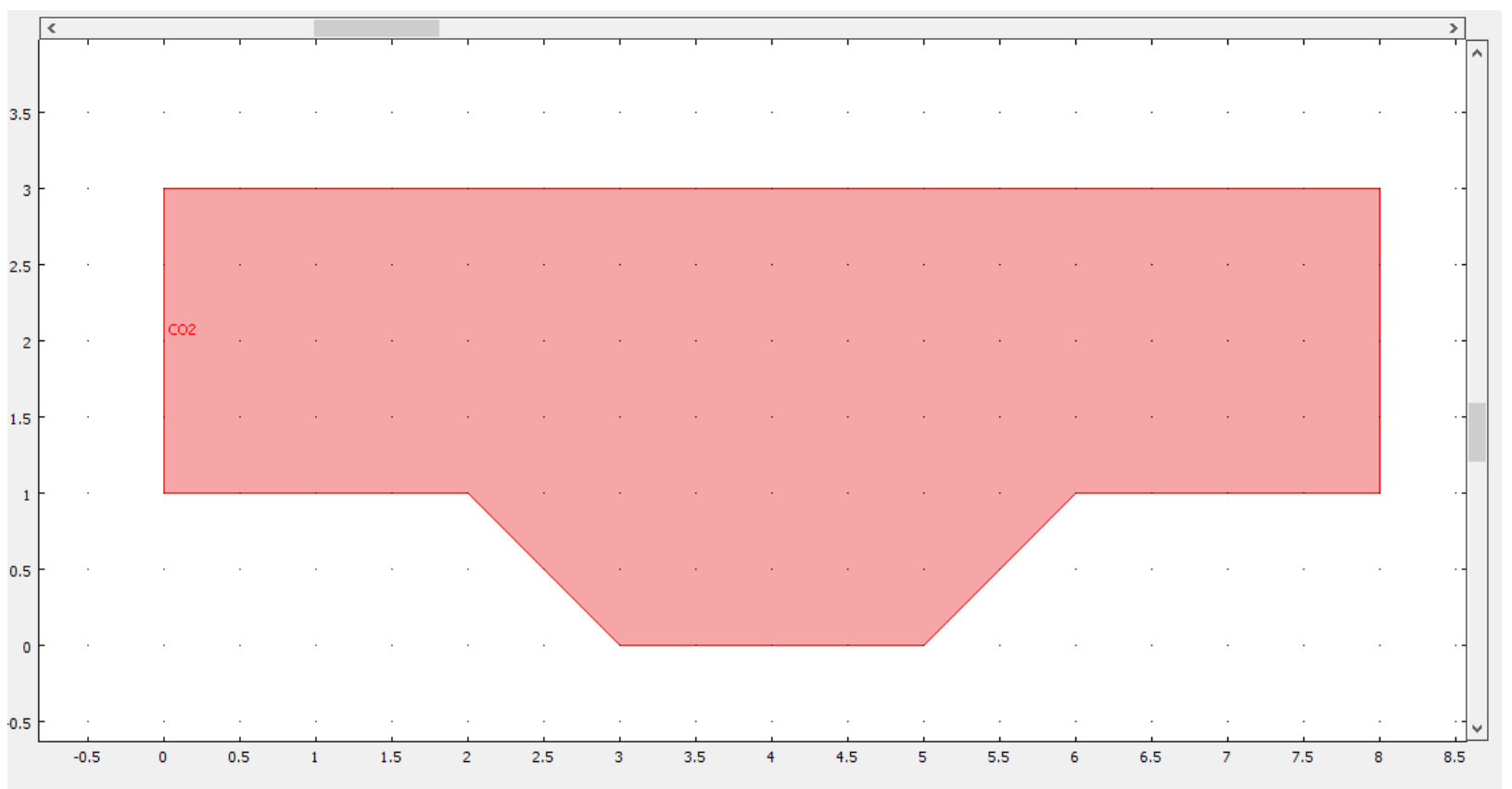

Рисунок 1 - Модель канала.

Систему дифференциальных уравнений запишем в виде:

$$
\rho u \cdot \nabla u=\nabla \cdot\left[-p I+\left(\eta+\eta_{T}\right)\left(\nabla u+(\nabla u)^{T}\right)\right]+F,
$$

$\nabla \cdot u=0$

$\rho u \cdot \nabla k=\nabla \cdot\left[\left(\eta+\frac{\eta_{T}}{\sigma_{k}}\right) \nabla k\right]+\eta_{T} P(u)-\rho \varepsilon$,

$\rho u \cdot \nabla \varepsilon=\nabla \cdot\left[\left(\eta+\frac{\eta_{T}}{\sigma_{\varepsilon}}\right) \nabla \varepsilon\right]+\frac{C_{\varepsilon 1} \varepsilon \eta_{T} P(u)-C_{\varepsilon 2} \rho \varepsilon^{2}}{k}$,

где

$$
\begin{aligned}
& P(u)=\frac{\nabla u}{\left(\nabla u+(\nabla u)^{T}\right)}, \\
& \eta_{T}=\frac{\rho C_{\mu} k^{2}}{\varepsilon},
\end{aligned}
$$

$\rho$ - плотность набегающего воздуха,

$\eta$ - динамическая вязкость в (Па·с) при заданной температуре $T$, [2-4]

$T$ - заданная температура в Кельвинах.

Граничные условия определим в виде:
- $\quad$ на входе потока:

$$
\begin{gathered}
u=-U_{0} n, \\
k=\frac{3\left(I_{T} U_{0}\right)^{2}}{2}, \\
\varepsilon=\frac{C_{\mu}^{0.75}\left(\frac{3\left(I_{T} U_{0}\right)^{2}}{2}\right)^{1.5}}{L_{T}},
\end{gathered}
$$

где $U_{0}$ - скорость набегающего потока.

- на выходе потока:

$$
\begin{aligned}
& \left(\eta+\eta_{T}\right)\left(\nabla u+(\nabla u)^{T}\right) n=0, \\
& p=p_{0}, \\
& n \cdot \nabla k=0, \\
& n \cdot \nabla \varepsilon=0 .
\end{aligned}
$$

Исследуемую модель канала (рис.1) разобьем на конечные элементы нерегулярной конечноэлементной сеткой (рис.2). 


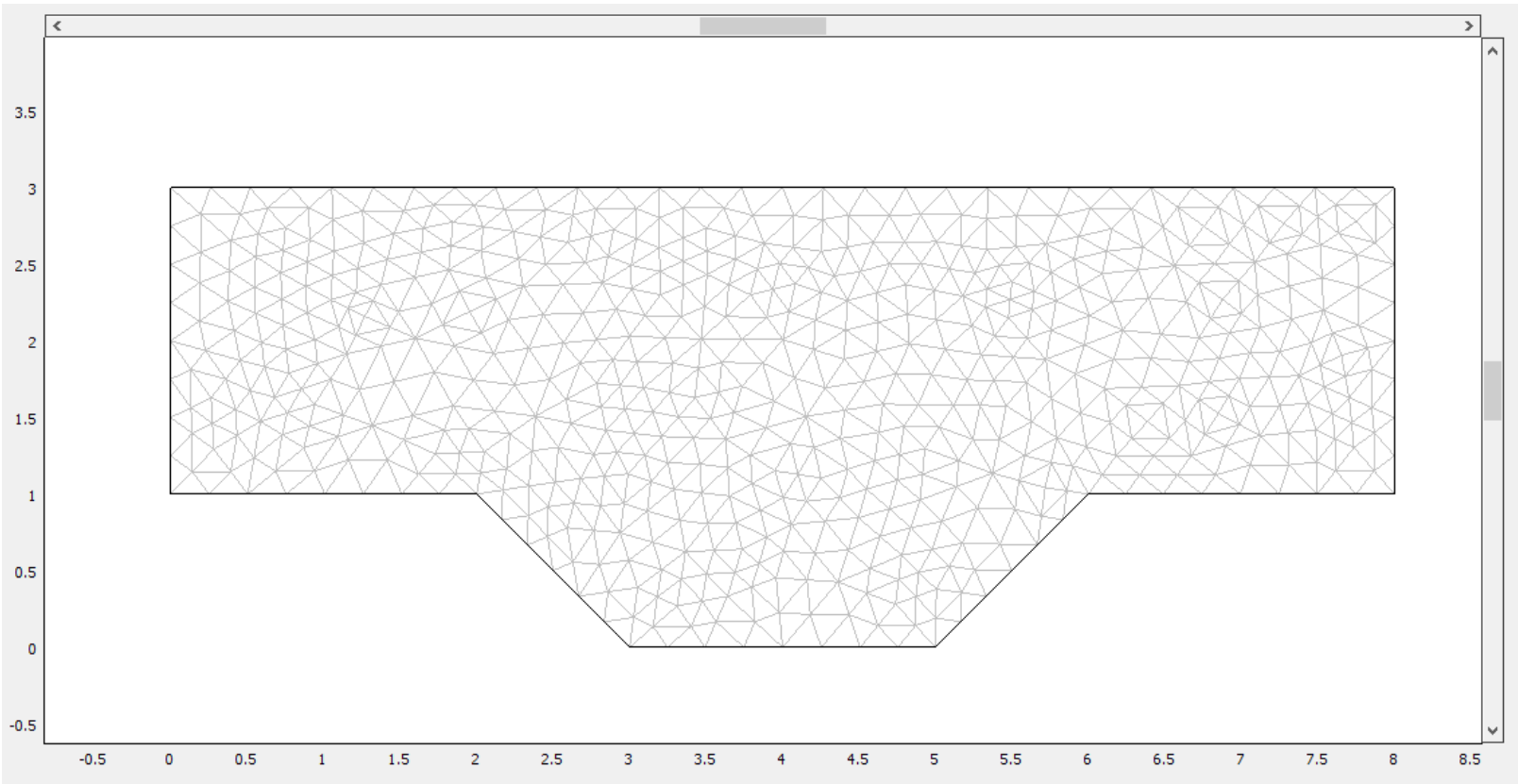

Рисунок 2 - Нерегулярная конечноэлементная сетка.

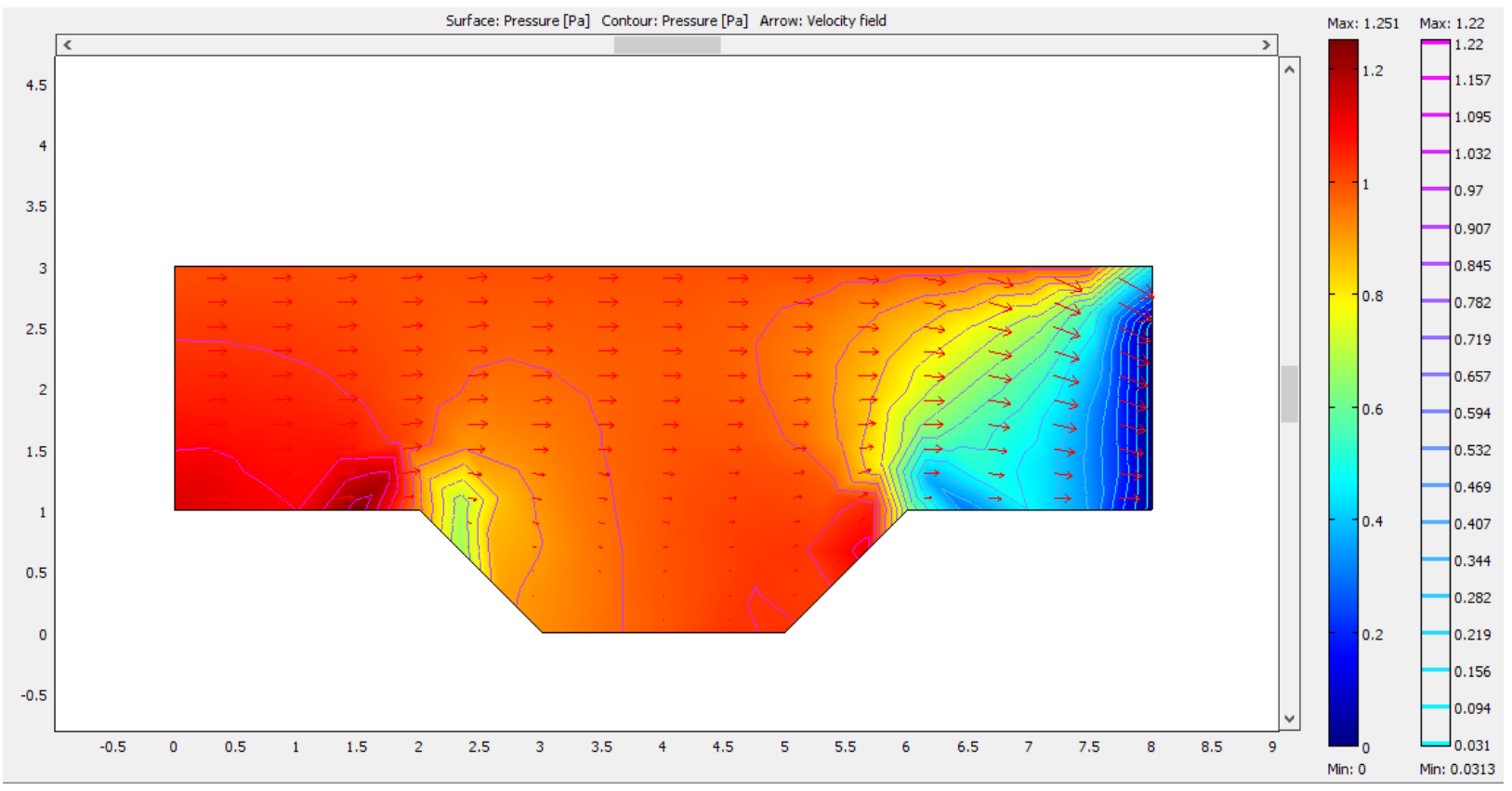

Рисунок 3 - Распределение давления воздуха и процесса аэродинамического обтекания пустого канала при скорости ветра 1 м/с.

На рис.3 контурными линиями изображены области повышенного давления. 


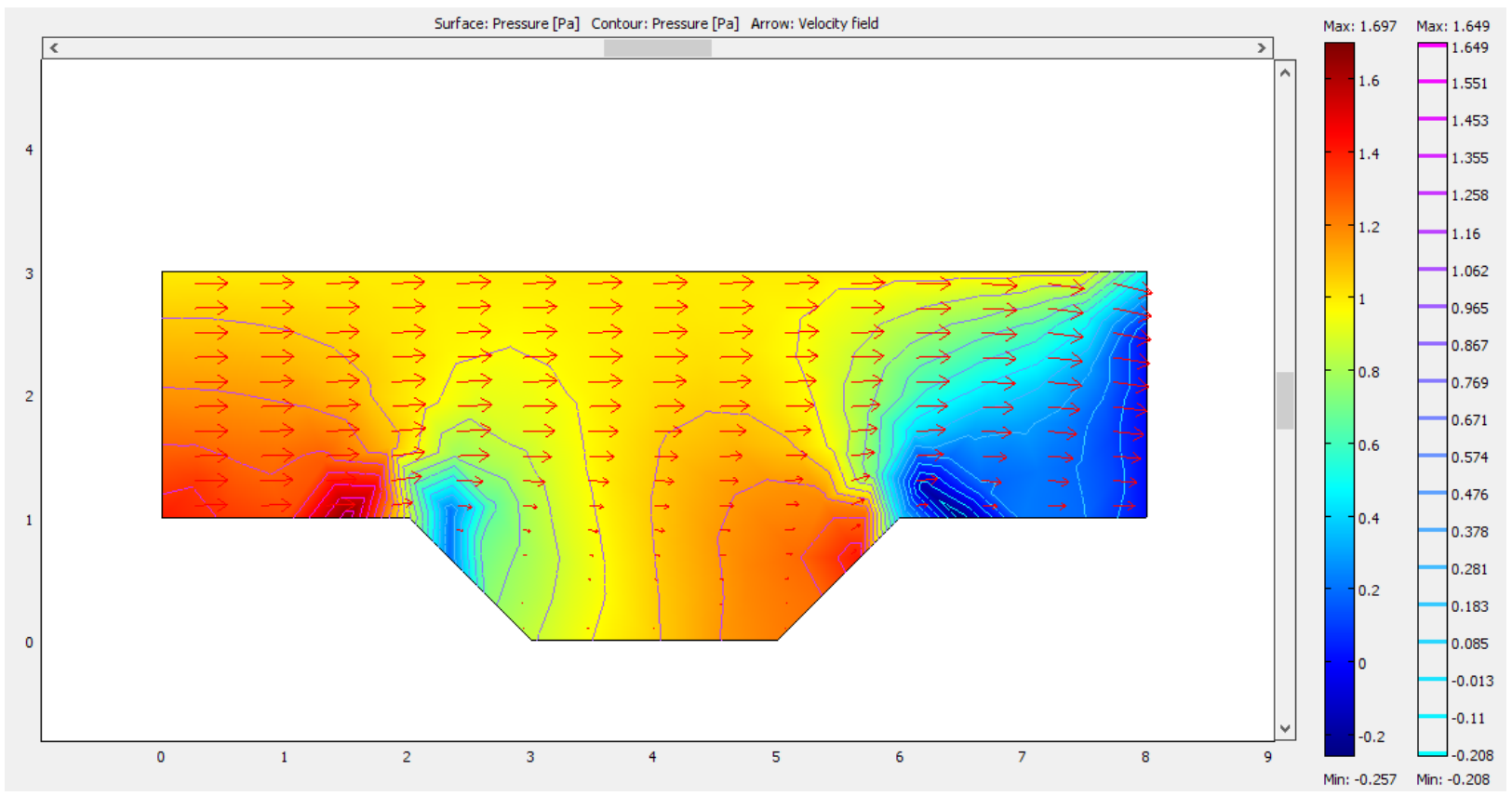

Рисунок 4 - Распределение давления воздуха и процесса аэродинамического обтекания пустого канала при скорости ветра 2 м/с.

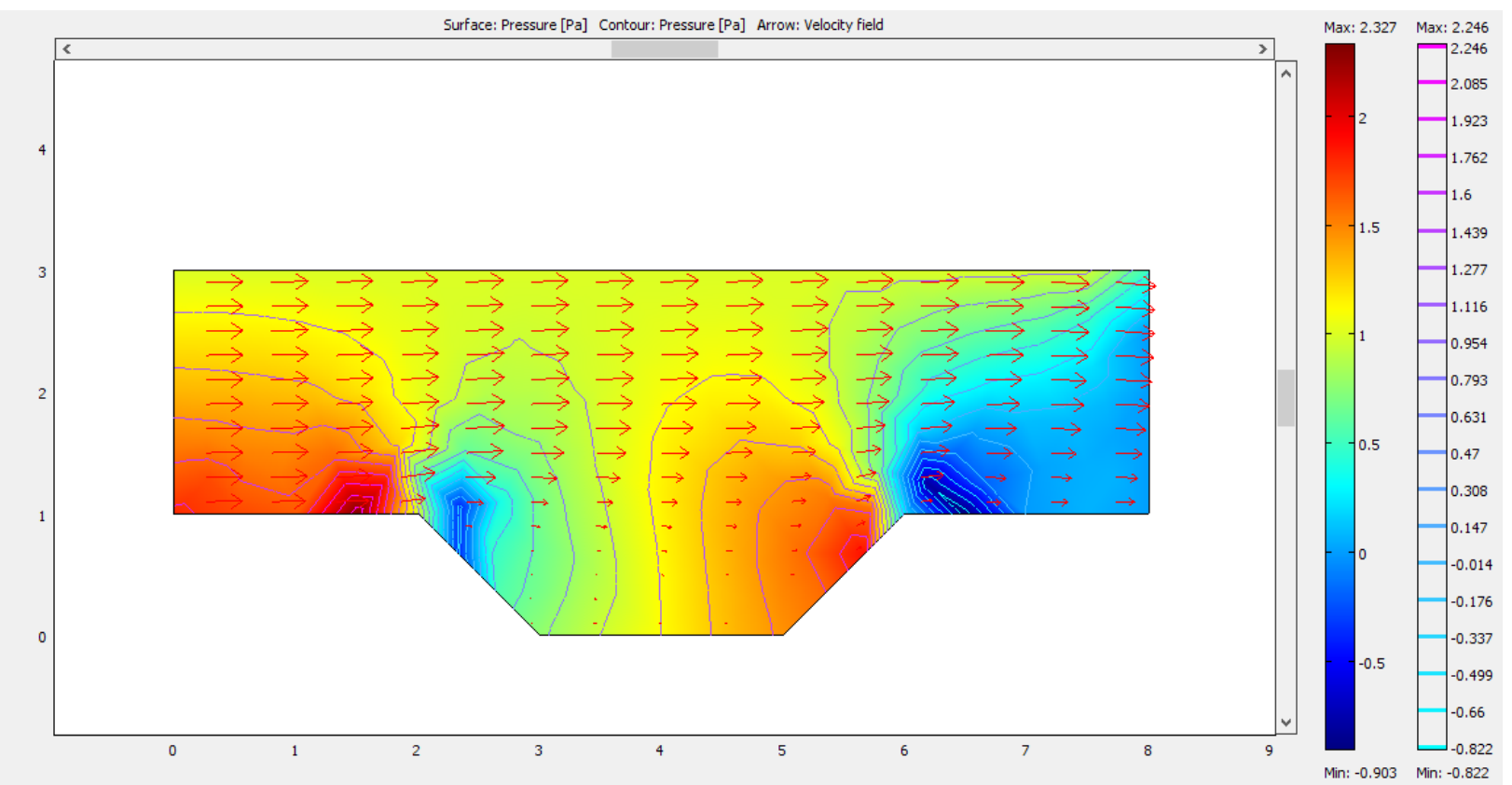

Рисунок 5 - Распределение давления воздуха и процесса аэродинамического обтекания пустого канала при скорости ветра 3 м/с. 


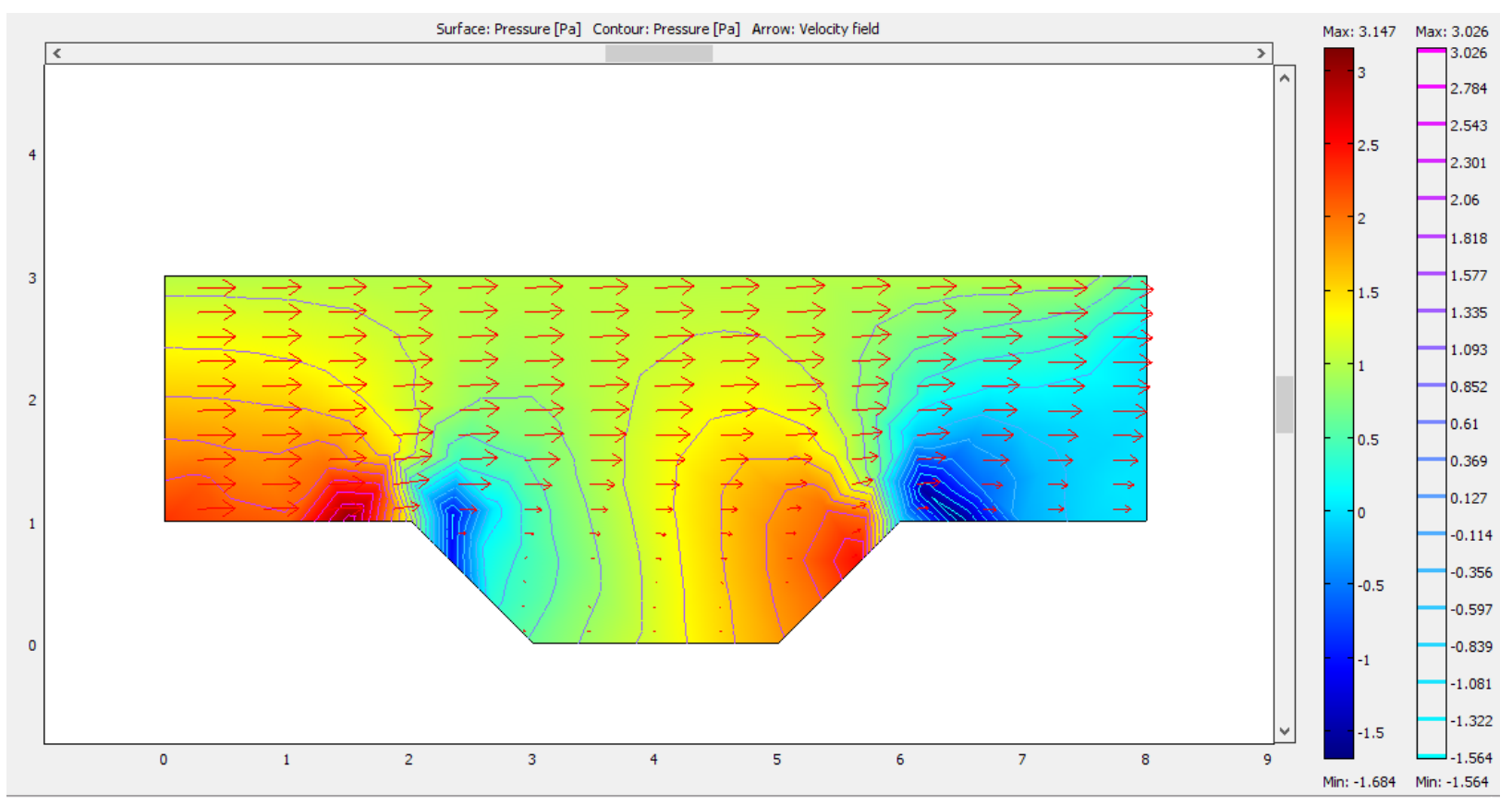

Рисунок 6 - Распределение давления воздуха и процесса аэродинамического обтекания пустого канала при скорости ветра 4 м/с.

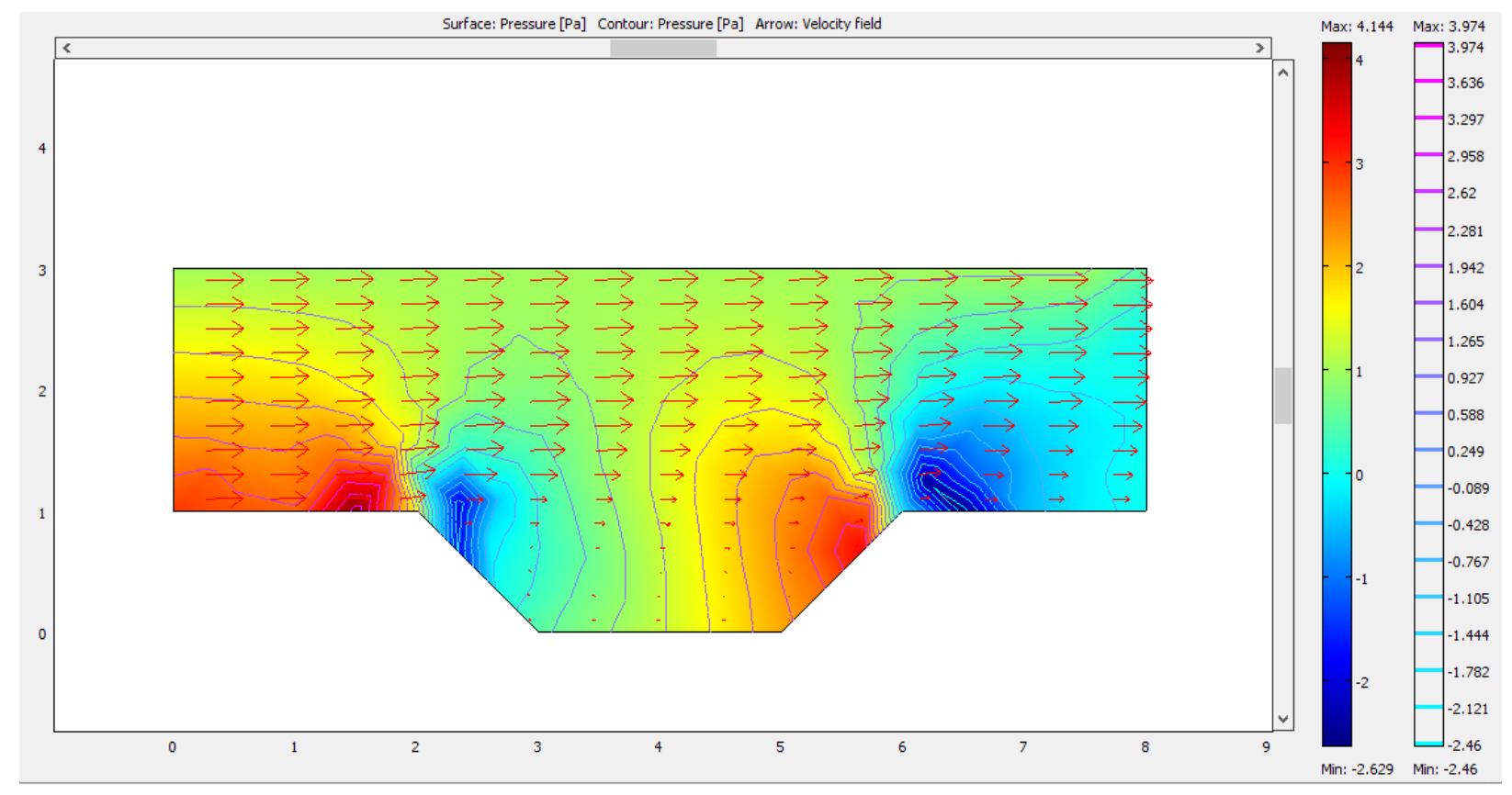

Рисунок 7 - Распределение давления воздуха и процесса аэродинамического обтекания пустого канала при скорости ветра 5 м/с. 


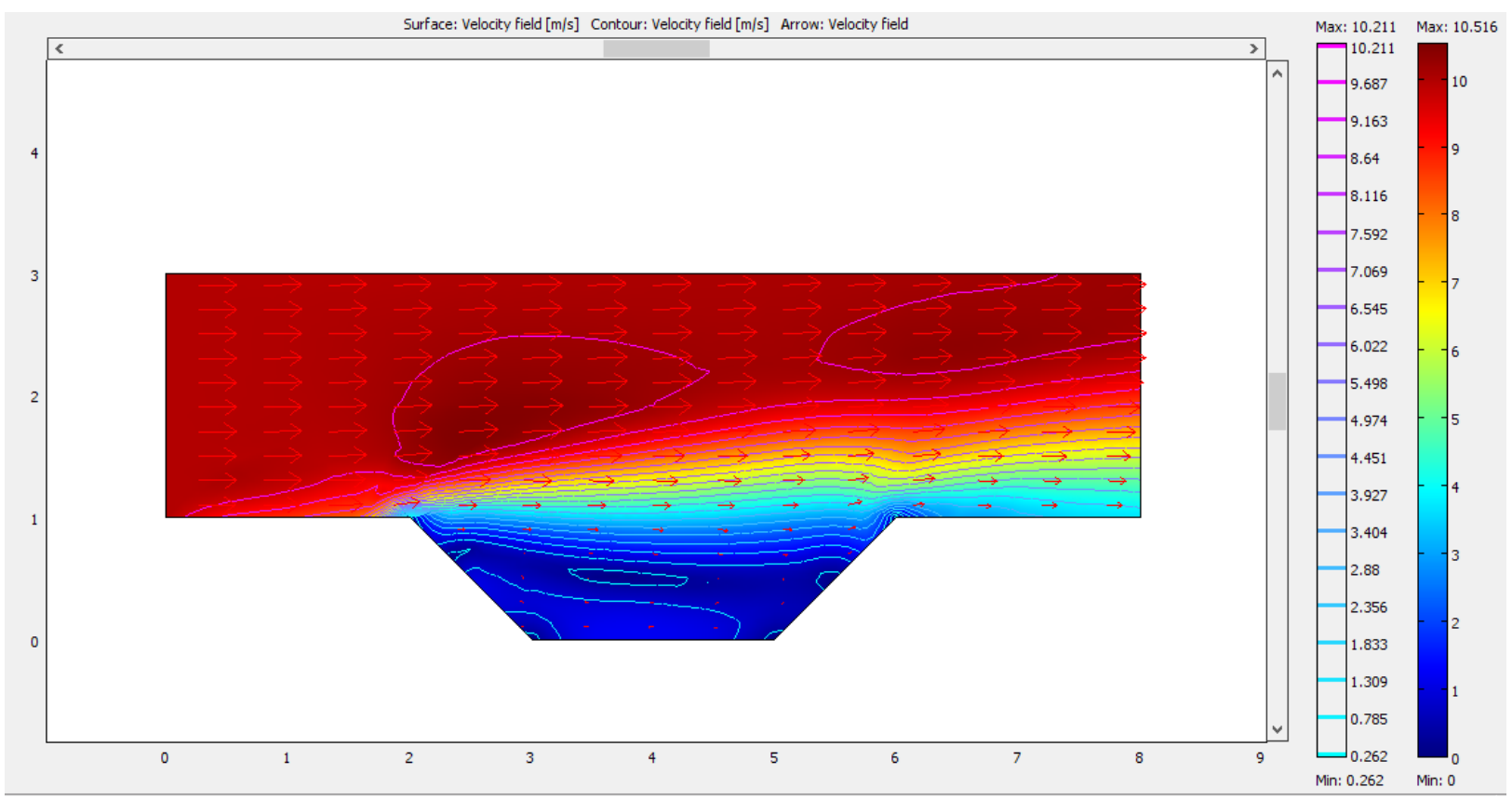

Рисунок 8 - Процессы аэродинамического обтекания пустого канала при порывах ветра до 10 м/с.

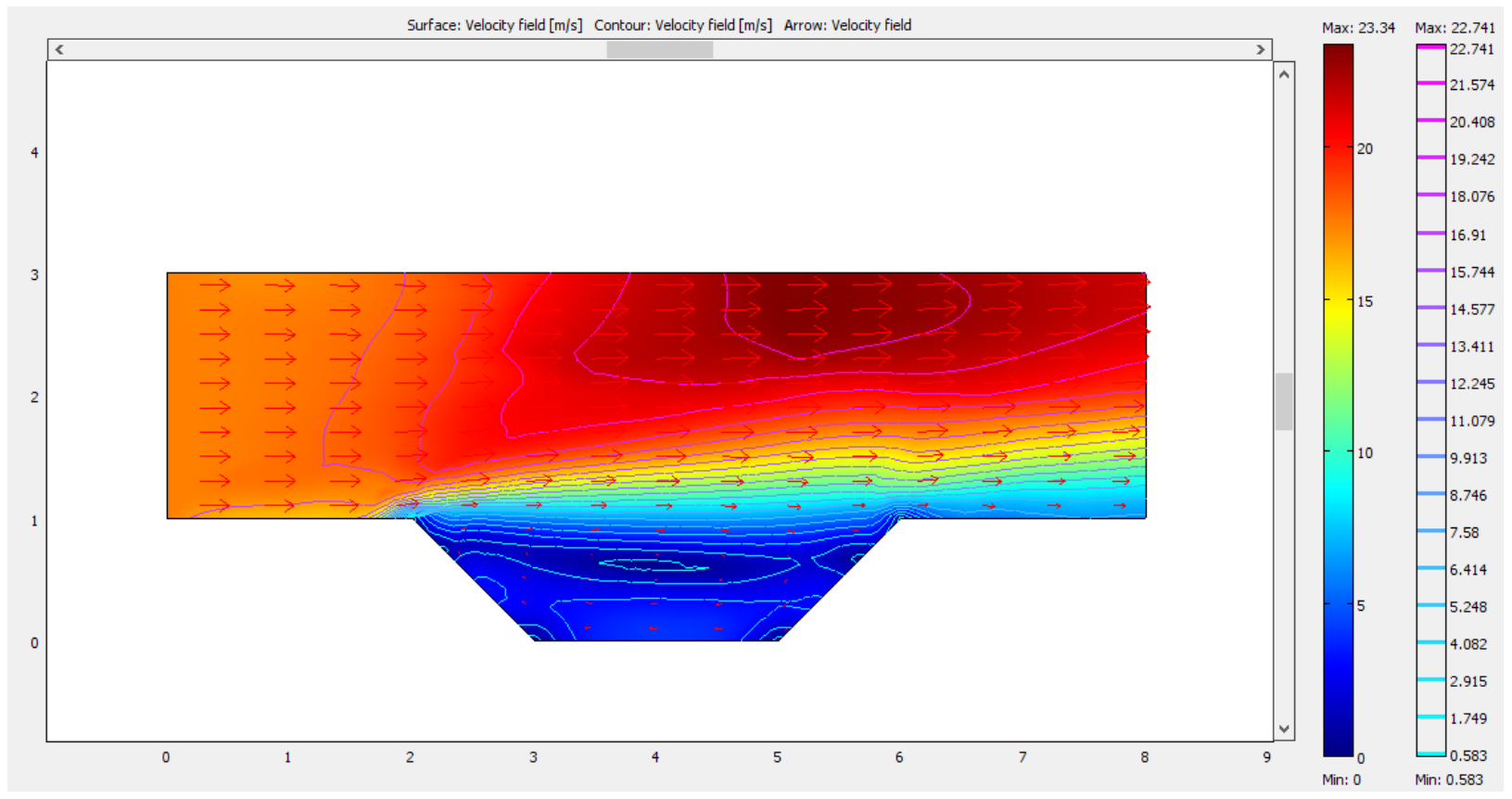

Рисунок 9 - Процессы аэродинамического обтекания пустого канала при порывах ветра до 20 м/с. 


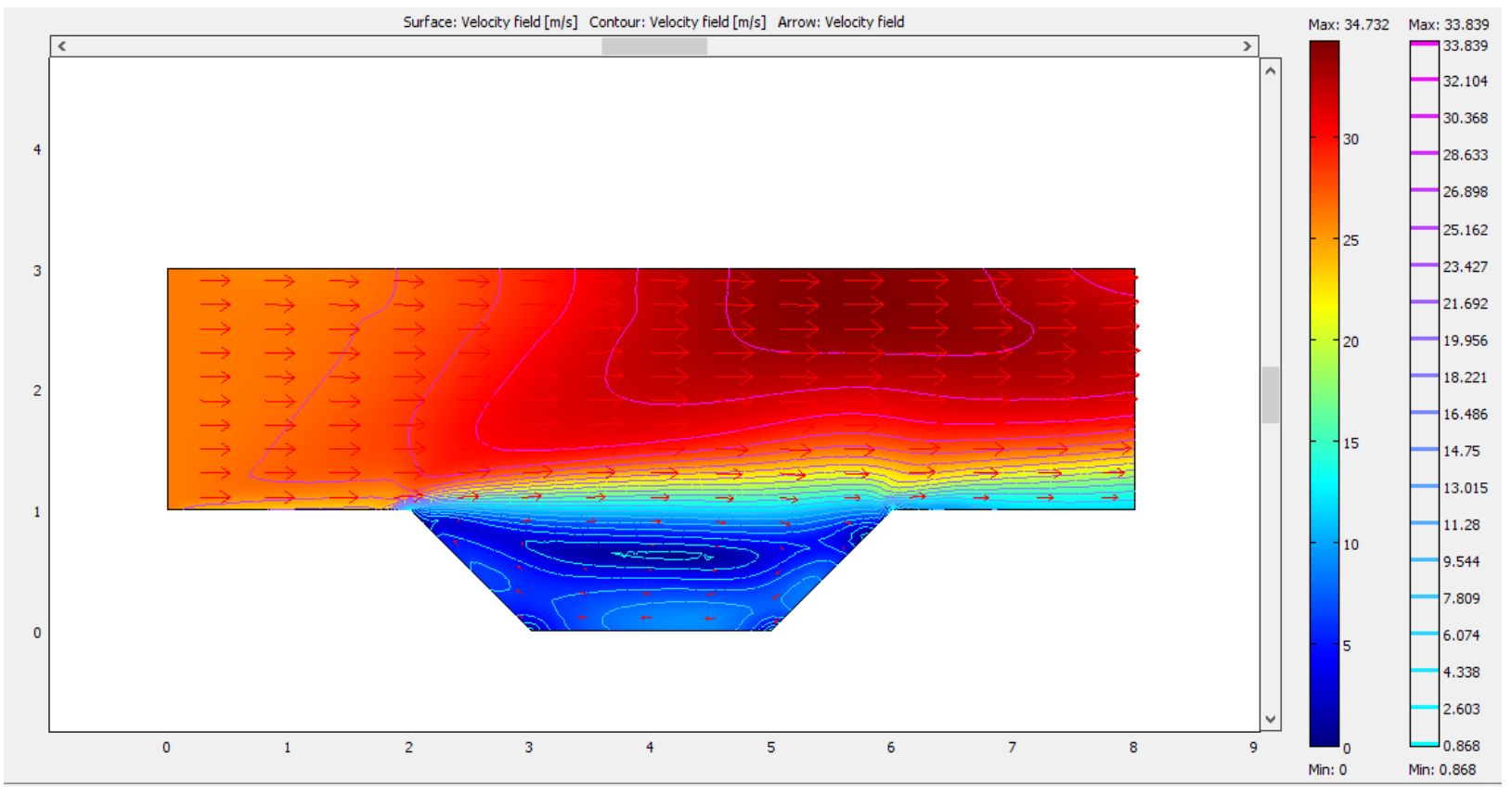

Рисунок 10 - Процессы аэродинамического обтекания пустого канала при порывах ветра до 30 м/с.

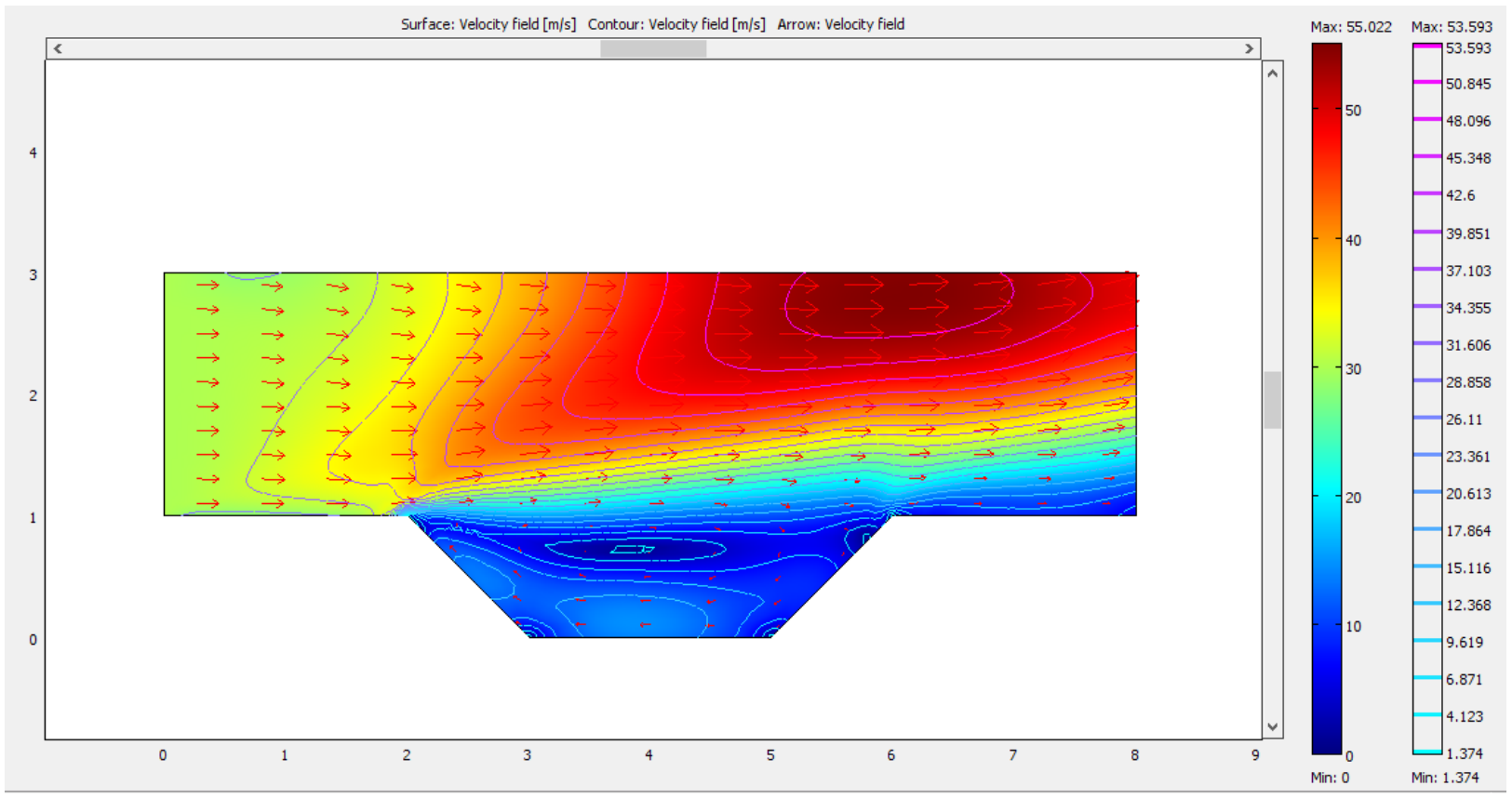

Рисунок 11 - Процессы аэродинамического обтекания пустого канала при порывах ветра до 40 м/с.

Выводы.

Построена математическая модель обтекания пустого канала. Получены аэродинамические характеристики процесса моделирования воздушного потока, при равномерном ветре, а также при порывах от 10 до 40 м/с. Полученные данные могут быть использованны при анализе и моделировании снегозаносимости и дефляции почв. 


\section{References:}

1. (1984) Ograzhdenie dlya zashchity ob"ektov ot snezhnykh zanosov. Patent № SU1237735A1.

2. (2015) Vyazkost'. Available: https://ru.wikipedia.org/wiki/ $\% \mathrm{C} 2 \% \mathrm{FF} \% \mathrm{E} 7 \% \mathrm{E}$ A \%EE $\% \mathrm{~F} 1 \% \mathrm{~F} 2 \% \mathrm{FC}$ (Accessed:25.01.15).

3. (2015) Dinamicheskaya vyazkost' gazov i parov. Available: http://thermalinfo.ru/publ/gazy/gazy raznye/din amicheskaja_vjazkost_gazov_i parov/29-1-0$\underline{316}$ (Accessed:25.01.15).

4. Koybakov S.M. (2013) Izobreteniya v oblasti gidrotekhniki i bezopasnosti zhiznedeyatel'nosti. -Taraz, TarGU, Taraz unversiteti, -2013, 112c.

5. (2015) Srednyaya skorost' vetra v regionakh Respubliki Kazakhstan. Available: http://www.energywind.ru/recomendacii/Skoros t-vetra-Kazakhstan (Accessed:25.01.15).

6. Zhunisbekov S, Koybakov SM, Shevtsov AN (2014) SOME FEATURES OF MATHEMATICAL MODELING OF COASTAL MANAGERITALIA. ISJ Theoretical \& Applied Science 07 (15): 36-42. doi:

http://dx.doi.org/10.15863/TAS.2014.07.15.6

7. Shevtsov AN, Alimbaev BA, Manapbaev BZ (2013) COMPUTER MODEL OF THE INFLUENCE OF MAGNETIC FIELDS OF THE DOMAINS IN THE FORM OF MICROCRACKS AT FRACTURE OF METALS. ISJ
Theoretical \& Applied Science 06 (2): 51-64. doi:

http://dx.doi.org/10.15863/TAS.2013.06.2.6

8. Shevtsov AN (2014) SOME QUESTIONS SIMULATION OF INTERACTIVE DYNAMIC SYSTEMS. ISJ Theoretical \& Applied Science $01 \quad$ (9): 5-22. doi: http://dx.doi.org/10.15863/TAS.2014.01.9.2

9. (2015) Raschet koeffitsienta dinamicheskoy vyazkosti gaza. Available: http://www.chemway.ru/bd chem/definition/me thods/desc_gas/mu calc.php

(Accessed:25.01.15).

10. (2015)Vikhrerazreshayushchee modelirovanie kak odin iz metodov opisaniya turbulentnykh techeniy. Available: http://www.inm.ras.ru/laboratory/direct2.htm (Accessed:25.01.15).

11. (2015) URAVNENIYa REYNOL"DSA DLYa OSREDNENNOGO TURBULENTNOGO DVIZhENIYa. Available:

http://studopedia.net/6 34965 uravneniyareynoldsa-dlya-osrednennogo-turbulentnogodvizheniya.html (Accessed:25.01.15).

12. Sugak EV, Sugak AV (2013) MODELIROVANIE TURBULENTNYKh ZAKRUChENNYKh POTOKOV. Available: http://www.science-education.ru/107-8312 (Accessed:25.01.15). 